\title{
Constraints on temporal variation of fundamental constants from GRBs
}

\author{
C. Bambi $^{1}$ and A. Drago ${ }^{2,3}$ \\ ${ }^{1}$ Department of Physics and Astronomy, Wayne State University, Detroit, MI 48201, USA \\ ${ }^{2}$ Istituto Nazionale di Fisica Nucleare, Sezione di Ferrara, I-44100 Ferrara, Italy \\ ${ }^{3}$ Dipartimento di Fisica, Università degli Studi di Ferrara, I-44100 Ferrara, Italy
}

(Dated: August 24, 2021)

\begin{abstract}
The formation of a strange or hybrid star from a neutron star progenitor is believed to occur when the central stellar density exceeds a critical value. If the transition from hadron to quark matter is of first order, the event has to release a huge amount of energy in a very short time, and we would be able to observe the phenomenon even if it is at cosmological distance far from us. Most likely, such violent quark deconfinement would be associated with at least a fraction of the observed gamma ray bursts. If we allow for temporal variations of fundamental constants like $\Lambda_{Q C D}$ or $G_{N}$, we can expect that neutron stars with an initial central density just below the critical value can enter into the region where strange or hybrid stars are the true ground state. From the observed rate of long gamma ray bursts, we are able to deduce the constraint $\dot{G}_{N} / G_{N} \lesssim 10^{-17} \mathrm{yr}^{-1}$, which is about 5 orders of magnitude more stringent than the strongest previous bounds on a possible increasing $G_{N}$.
\end{abstract}

INTRODUCTION - Search for temporal variation of "fundamental constants" has attracted a lot of interest in last years [1]. Such a phenomenon is quite a general prediction of many different frameworks (superstring theories, scalar-tensor theories, models in extra dimensions, etc.) when the dynamics of the whole universe is taken into account and a clear sign of violation of the Strong Equivalence Principle (SEP) 2]. On the other hand, the Einstein Equivalence Principle (EEP) - which is the essence of the geometrical theory of gravitation refers only to non-gravitational physics and thus allows the Newton constant $G_{N}$ to be time dependent, but still forbids any temporal variation of non-gravitational parameters $\left(\alpha, \Lambda_{Q C D}, G_{F}\right.$, etc. $)[2]$.

Since quantitative predictions are impossible at present, the common approach is based on model independent investigations of possible temporal evolution of physical quantities that should instead be constant in the standard theory and constraints are often reported assuming variations that are linear in the cosmological time: indeed one can expect to expand any false constant in a power series of time and that the linear term is the leading-order correction. For this reason, essentially all the works in the literature on this subject do not specify any theoretical framework and the discussion is at a pure phenomenological level. However, this approach cannot be always justified and hence, in general, one cannot directly compare bounds obtained today in laboratory with others coming from arguments involving the physics of the early universe.

The topic of time varying "fundamental constants" has received additional interest after the claims of some evidence of time variation of the fine structure constant $\alpha[3]$ and of the proton to electron mass ratio $m_{p} / m_{e}[4]$. There are indeed good theoretical arguments to believe that a possible temporal evolution of $\alpha$ implies time shift for other quantities as well [5]. However the observational situation is quite controversial, since other groups have not confirmed the results [6] .

A relevant quantity which sets strong interactions and hadron masses is the QCD scale $\Lambda_{Q C D}$. The most stringent constraint on its possible temporal variation comes from an analysis of isotopic abundances of the Oklo uranium mine in Gabon, a natural fission reactor that operated about $2 \cdot 10^{9}$ years ago for about $10^{5}$ years. Assuming a linear time dependence, one gets [7]

$$
\left|\dot{\Lambda}_{Q C D} / \Lambda_{Q C D}\right| \lesssim 10^{-17} \mathrm{yr}^{-1}
$$

Other constraints on $\dot{\Lambda}_{Q C D}$ are usually quite model dependent and, in any case, at least 2 orders of magnitude weaker than the bound in (11).

However, among all the "fundamental constants" a privileged role is played by the gravitational constant $G_{N}$, whose variation violates only the SEP. The tightest constraint in the literature on a linear temporal evolution of $G_{N}$ is provided by the Lunar Laser Ranging experiment, which has monitored Earth-Moon distance for about 30 years. The result is [8]

$$
\dot{G}_{N} / G_{N}=(4 \pm 9) \cdot 10^{-13} \mathrm{yr}^{-1} .
$$

On the other hand, the earliest event in the history of the universe which has left a reliable record of the past value of $G_{N}$ is the Big Bang Nucleosynthesis: from the primordial abundance of ${ }^{4} \mathrm{He}$ we can conclude that, when the universe was only 1 s old, i.e. $\sim 13 \mathrm{Gyr}$ ago, $G_{N}$ could not differ more than about $10 \%$ from its present value [9]. Other interesting constraints on $\dot{G}_{N} / G_{N}$, at the level of $10^{-12} \mathrm{yr}^{-1}$ in the case of linear time evolution, can be deduced for instance from the orbital period of binary pulsars [10], from the comparison of masses of old and young neutron stars [11] and from the so called gravitochemical heating in neutron stars [12].

In this letter, we consider the effects of a temporal variation of $\Lambda_{Q C D}$ and $G_{N}$ on the equilibrium configuration of compact stars: indeed, non-zero $\dot{\Lambda}_{Q C D}$ or $\dot{G}_{N}$ could change the true ground state of some stars and induce conversion of neutron stars into strange or hybrid ones. Here the keypoint is that the transformation probably 
involves a first order phase transition, leading to the release of a large amount of energy in a short time. This makes even small variation of $\Lambda_{Q C D}$ or $G_{N}$ to produce spectacular events, observable at cosmological distances. Taking into account the possible events which could be somehow associated with these phenomena, we can estimate an upper limit for the decreasing rate of $\Lambda_{Q C D}$ or for the increasing rate of $G_{N}$.

To avoid any possible misunderstanding, a comment is in order here; we can only constrain dimensionless quantities, such as $\alpha$. When we talk about temporal evolution of dimensionful constants, like $\Lambda_{Q C D}$ and $G_{N}$, we implicitly assume a particular system of units and again we constrain the dimensionless ratio to standards chosen as units and constant by definition [1]. This is particularly evident for example in some scalar-tensor theories of gravity, where one can work in the Einstein frame (where $G_{N}$ is constant) or in the Jordan frame (where particle masses are constant and $G_{N}$ can change), see e.g. Ref. [13]. However, this is a more general result and one can always choose an arbitrary dimensionful quantity as standard unit and then compare it with other quantities. And this is basically also what we do here: when we assume $G_{N}$ constant, we allow for variation of $\Lambda_{Q C D}$, and when we assume $\Lambda_{Q C D}$ constant we discuss the possibility of non-zero $\dot{G}_{N}$, but we compare always the QCD interaction with the gravitational one. For this reason, the possibility of a compensation between the temporal variation of the QCD energy scale $\Lambda_{Q C D}$ and of the gravitational energy scale $M_{P l}=G_{N}^{-1 / 2}$ has no physical meaning: only their dimensionless ratio is an observable. Of course, here we cannot exclude the possibility that both $\Lambda_{Q C D}$ and $M_{P l}$ change exactly in the same way with respect to another dimensionful constant, for example the vacuum expectation value of the Higgs field $v$, but such a case sounds quite $a d h o c$ and would require an unwanted fine-tuning.

COMPACT STARS - Compact stars are the end-product of heavy stars after supernova explosion [14, 15]. Their mass is typically $\sim 1.5 M_{\odot}$ and their radius is about 10 $\mathrm{km}$, so that they are extremely dense objects: in particular we expect that the central baryon density, $\rho_{c}$, is at the level of $2-10$ times normal nuclear matter density $\rho_{0}=0.16 \mathrm{fm}^{-3}$. At such high densities, matter would be made of neutrons, with a smaller fraction of protons and electrons. However, in the upper region of this interval, the ground state is probably represented by strange matter of $u, d$ and $s$ deconfined quarks: the latter are fermions and introducing a third flavor there are new low energy available states which can reduce the total energy of the system. If so, compact stars with a central density higher than a critical value $\rho_{\text {crit }}$ would be strange stars (stars made entirely of strange matter) or hybrid stars (stars with a strange matter core and an outer part of hadron matter), depending on QCD physics and/or their mass. For a review, see e.g. Ref. [15]. Thanks to these extreme conditions, impossible to reach in laboratories on the Earth, compact stars can really be seen as a unique opportunity to investigate new physics [16, 17].

The results of this letter are essentially based on the following two assumptions:

1. Strange or hybrid stars exist, but not all the compact stars are strange or hybrid.

2. The transition from hadron to strange matter is of first order.

The first statement means that we assume that the population of compact stars is made of both neutron and strange/hybrid stars. In other words, the critical density $\rho_{\text {crit }}$ has to be in the range of the typical central density of compact stars, so that the ones with $\rho_{c}<\rho_{\text {crit }}$ are neutron stars, while if $\rho_{c}>\rho_{\text {crit }}$ they are strange or hybrid. As for the second hypothesis, it is indeed what is commonly believed, i.e. at high baryon density the phase transition from hadron to strange matter seems to be of first order [18]. Unfortunately, up to now no model independent argument supporting that hypothesis has been provided.

Let us now introduce the information which is needed to constrain the temporal variation of the QCD scale and of the gravitational constant. From considerations on stellar evolution, we can say that about $0.1 \%$ of the stars in galaxies are compact ones, that is neutron or strange stars (see e.g. Ref. [14]). This implies that there are about $10^{8}$ compact stars in a typical galaxy and that the whole visible universe contains a total number of $N_{\text {tot }} \sim 10^{20}$ [27]. As for their central baryon density $\rho_{c}$, we assume for simplicity that compact stars are uniformly distributed with $\rho_{c}$ in the range $\left(2 \rho_{0}, 10 \rho_{0}\right)$, that is the central density distribution is

$$
n\left(\rho_{c}\right) \approx \frac{N_{t o t}}{8 \rho_{0}}
$$

for $2 \rho_{0}<\rho_{c}<10 \rho_{0}$ and 0 otherwise. Here we would like to stress that this simple choice is not crucial for our conclusions. Indeed, we could consider another distribution and get roughly the same result if the probability of finding a star with a given central density is the same within an order of magnitude: for example, Eq. (3) could be seen as the approximation of a gaussian of height $\sim N_{\text {tot }} / 8 \rho_{0}$ and width $\sim 8 \rho_{0}$. Following our first working hypothesis, $2 \rho_{0}<\rho_{\text {crit }}<10 \rho_{0}$ and the possibility of temporal variation of $\Lambda_{Q C D}$ and $G_{N}$ may induce the transformation of neutron stars into strange or hybrid ones, because $\rho_{\text {crit }}$ depends on $\Lambda_{Q C D}$ and $\rho_{c}$ on $G_{N}$. In addition to this, since we take the phase transition to be of first order, in the conversion the compact object has to release a huge amount of energy in a very short time, say $10 \mathrm{~s}$. This energy is the difference of binding energy between strange/hybrid star and neutron one. All the models agree with a typical energy release at the level of $10^{52}-10^{53} \mathrm{erg}[19]$. This huge amount of energy can be converted, at least in part, to photons and $e^{+} e^{-}$pairs. Various mechanisms have been proposed [20], all of them 
producing a gamma emission with an energy easily exceeding $10^{50}-10^{51} \mathrm{erg}$. Such a huge power in $\gamma$ can be at the origin of at least a fraction of the so called long Gamma Ray Bursts (GRBs) [21].

Temporal variation of $\Lambda_{Q C D}$ - A temporal variation of $\Lambda_{Q C D}$ must induce a change in the value of the critical baryon density $\rho_{\text {crit }}$. A rough estimate can be obtained as follows. We start by considering two types of quark models, the bag-like [22] and the NJL-like models [23]. In bag models, the crucial parameter regulating $\rho_{\text {crit }}$ is the pressure of the vacuum $B$, whose numerical value is independent of the presence of the quarks. Therefore the value of $B$ is controlled by the only dimensional parameter present also in pure gauge QCD, i.e. $\Lambda_{Q C D}$. One can therefore write

$$
\rho_{\text {crit }}=a \Lambda_{Q C D}^{4},
$$

where $a$ is a dimensionless order one coefficient. In chiral models, and in particular in the NJL-like ones, gluons are not present as dynamical degrees of freedom. It is anyway possible to introduce a quantity whose physical meaning is similar to the one of $B$ in bag models (see e.g. Ref. [23]). The numerical value of that quantity is dominated by the value of the constituent masses of the quarks. In NJL-like models the mass of the strange quark is so large that it plays a minor role compared to the up and down quarks. Since the constituent masses of the $u$ and $d$ quarks are almost independent of the small value of the current mass, one can conclude that also in chiral models the "pressure of the vacuum" depends almost only on $\Lambda_{Q C D}$.

In the bag model, the relation between $\rho_{\text {crit }}$ and $B$ has been evaluated in a large number of calculations and is typically of the order of [15, 24]

$$
\Delta \rho_{\text {crit }} \approx \frac{0.003}{\mathrm{MeV}} \Delta B
$$

where $\Delta \rho_{\text {crit }}$ and $\Delta B$ are respectively a variation of $\rho_{\text {crit }}$ and $B$. From Eq. (41) we find that $\Delta B / B=$ $4 \Delta \Lambda_{Q C D} / \Lambda_{Q C D}$ and hence

$$
\Delta \rho_{\text {crit }} \sim 10 \rho_{0} \Delta \Lambda_{Q C D} / \Lambda_{Q C D} .
$$

Let us note that $\Delta \Lambda_{Q C D}<0$ implies $\Delta \rho_{\text {crit }}<0$ : a time decreasing $\Lambda_{Q C D}$ makes $\rho_{\text {crit }}$ decrease as well and neutron stars with a central density just below the critical value may convert into strange or hybrid ones. The rate of strange/hybrid star production due to the possible temporal evolution of $\Lambda_{Q C D}$ we can observe is

$$
\dot{N}_{s s} \sim-\epsilon n\left(\rho_{c}\right) \dot{\rho}_{c r i t} \sim-10^{20} \epsilon \frac{\dot{\Lambda}_{Q C D}}{\Lambda_{Q C D}} .
$$

Here $\epsilon$ is the fraction of compact stars in the visible universe whose conversion from hadron to strange matter can be seen from the Earth and the equation is valid only for $\dot{\rho}_{c}<0$ (hence $\dot{N}_{s s}>0$ ). If $\dot{\rho}_{c}>0$, strange stars should convert into neutron ones, but in this case we do not expect any violent phenomenon: more probably the picture would look like a slow evaporation of strange matter into hadrons. Because of this, no bound can be deduced for the case $\dot{\Lambda}_{Q C D}>0$.

In conclusion, according to our theoretical framework, a time decreasing $\Lambda_{Q C D}$ must be accompanied by spectacular events, that is the formation of strange or hybrid stars from neutron star progenitors. As already mentioned, the only known events which can be associated to such violent phenomena are the GRBs 21]. In particular we are interested in the so-called long GRBs, which are known to be associated with the gravitational collapse of massive stars. Here we would like to stress that we are not assuming that all or some of the long GRBs are related to the formation of a strange star, but we are saying that the conversion from a neutron to a strange star, if it occurs in the universe, is certainly a quite particular event which likely releases a huge amount of energy $\left(\sim 10^{52} \mathrm{erg}\right)$ in a short time and that at present we have not yet observed other phenomena compatible with this picture but long GRBs. From this point of view, we can assert that the rate of strange star production cannot exceed the one of GRBs. Since today the observed GRBs have a mean redshift $z \approx 3$ and are more or less uniformly distributed up to redshift $z \approx 5$, we would be able to observe most of the events in the whole visible universe associated with the transition from a neutron star to a strange star. This means that we can put $\epsilon \sim 1$ in Eq. (77). In the end, since the observed rate of GRBs is

$$
\dot{N}_{G R B s} \approx 10^{3} \mathrm{yr}^{-1},
$$

we deduce the following constraint on a possible linearly time decreasing QCD scale [28]

$$
-\dot{\Lambda}_{Q C D} / \Lambda_{Q C D} \lesssim 10^{-17} \mathrm{yr}^{-1} .
$$

One caveat is in order. If the transition from a neutron star to a hybrid or a quark star produces a beamed emission, the actual limit on $\dot{N}_{G R B s}$ is larger. GRBs are known to be beamed, and the correction to the rate due to beaming is of the order of $75 \pm 25$ [25]. If the same beaming factor applies to GRBs associated with quark deconfinement, than $\dot{N}_{G R B s} \approx 10^{5} \mathrm{yr}^{-1}$ and $-\dot{\Lambda}_{Q C D} / \Lambda_{Q C D} \lesssim 10^{-15} \mathrm{yr}^{-1}$.

TEMPORAL VARIATION OF $G_{N}$ - Let us now consider the possibility of a non-zero $\dot{G}_{N}$. Now the critical density $\rho_{\text {crit }}$ is unchanged, because it is set by QCD physics. However, like all the other stars, neutron stars are selfgravitating system whose configuration is determined by the balance between the attractive gravitational force and the particle pressure. Hence a variation of $G_{N}$ induces a change of the central stellar density. Of course, if a neutron star has a central density just below the critical value, an increase of $G_{N}$ makes $\rho_{c}$ pass the critical value and the neutron star can convert into a strange one. A rough estimate for the bound on $\dot{G}_{N} / G_{N}$ can be deduced from the following simple considerations. As 
stellar model, we can take a system of $N$ non-relativistic fermions of mass $m$ which interact only gravitationally. The energy per unit volume is

$$
\varepsilon \sim \frac{3}{5} \frac{p_{F}^{2}}{2 m} \rho+m \rho-\frac{3}{5} \frac{G_{N} m^{2} \rho^{2} V}{R},
$$

where $\rho$ is the particle number density (for the sake of simplicity we assume it is constant in the star), $p_{F}=$ $\left(3 \pi^{2} \rho\right)^{1 / 3}$ is the Fermi momentum, $R$ the stellar radius and $V$ the stellar volume. The first term on the right hand side of Eq. (10) is the particle kinetic energy per unit volume, the second term is the particle rest energy per unit volume and the last one is the gravitational potential energy per unit volume. If we multiply Eq. (10) by $1 / \rho$ and then we replace $\rho$ with $N / V$, we get the energy per particle as a function of the stellar radius $R$, since $N$ must be constant. It is straightforward to find that the equilibrium radius is proportional to $1 / G_{N}$. In the Newtonian framework, for a star with constant density we have $\rho \propto R^{-3} \propto G_{N}^{3}$. At first approximation, we can expect the same dependence of the central density of compact stars on the gravitational constant $G_{N}$ and the relation between a small change of $G_{N}$ and $\rho_{c}$ is

$$
\Delta \rho_{c} / \rho_{c} \approx 3 \Delta G_{N} / G_{N} .
$$

Hence, replacing $\dot{\rho}_{\text {crit }}$ with $-\dot{\rho}_{c}$ in Eq. (7), we find the bound on the temporal evolution of $G_{N}$

$$
\dot{G}_{N} / G_{N} \lesssim 10^{-17} \mathrm{yr}^{-1} .
$$

As we have already said for the case of the QCD scale $\Lambda_{Q C D}$, such a bound is applicable only in one direction, here for an increasing gravitational constant. However, Eq. (12) is about 5 order of magnitude more stringent than the present best constraint of Eq. (2). Also in this case, if the gamma emission is beamed the limit becomes less stringent, $\dot{G}_{N} / G_{N} \lesssim 10^{-15} \mathrm{yr}^{-1}$.

CONCLUSION - In this letter we have discussed the possibility that the QCD scale $\Lambda_{Q C D}$ is decreasing in time or that the Newton gravitational constant $G_{N}$ is increasing, considering their effects on the equilibrium configuration of compact stars. Under a set of reasonable assumptions, we have found the constraints

$$
\begin{aligned}
-\dot{\Lambda}_{Q C D} / \Lambda_{Q C D} & \lesssim 10^{-17} \mathrm{yr}^{-1}, \\
\dot{G}_{N} / G_{N} & \lesssim 10^{-17} \mathrm{yr}^{-1},
\end{aligned}
$$

for the case of linearly time varying $\Lambda_{Q C D}$ and $G_{N}$. Since these bounds come from the non-observation of too much GRBs, they cover the red-shift range $z \approx 0-5$, that is a time interval of about $10^{10}$ years.
The possibility of time varying "fundamental constants" is not exotic, but instead quite a general prediction of most theories of gravity beyond general relativity. Assuming that compact stars can be neutron or strange/hybrid stars, depending on the value of their central density, we have to expect that $\dot{\Lambda}_{Q C D}<0$ or $\dot{G}_{N}>0$ induces the transformation of some neutron stars into strange or hybrid ones. Then, if the phase transition from hadron to quark matter at such high density is of first order, as it is commonly believed, the energy release is huge and we should be able to observe the event even if it is at cosmological distant far from us. At present, the only phenomena compatible with this picture are the GRBs and, from their rate, we arrive at the constraints on the temporal evolution of $\Lambda_{Q C D}$ and $G_{N}$. On the other hand, if the origin of GRBs were completely different, it would mean that at present we do not know any phenomenon which can be associated to the formation of a strange/hybrid star from a neutron star and our bounds would become much stronger. Of course, it is important that future investigations will confirm our working hypothesis. In the end, we would like to stress that our constraints (9) and (12) (or (13) and (14)) are very competitive, even from the point of view of the covered time interval (at present, only BBN and CMBR arguments can probe earlier events in the history of the universe). In particular for the case of the gravitational constant, our bound on a linear time increasing $G_{N}$ is much tighter than all the previous bounds, even if a possible beaming of the GRB associated with quark deconfinement is taken into account. On top of that, the previous bounds will be hardly significantly improved in a near future for the coming of new measurements and observations. On the contrary, our constraint could become much stronger once the origin of GRBs will be better known.

Finally, it is tempting to discuss a recent analysis indicating an excess of long GRBs at large red-shift [26]. More precisely, an evidence has been found of a GRB rate 4 times larger than the one predicted assuming the GRB rate to follow the star formation rate. A possible interpretation of this result can be based on a non-linear evolution of the fundamental constants, with a more rapid variation in the young universe.

\section{Acknowledgments}

We wish to thank Alexey Petrov for useful comments. C.B. is supported in part by NSF under grant PHY-0547794 and by DOE under contract DE-FG0296ER41005.
[1] J.P. Uzan, Rev. Mod. Phys. 75, 403 (2003) arXiv:hep-ph/0205340.
[2] C.M. Will, Living Rev. Rel. 9, 3 arXiv:gr-qc/0510072.
$(2006)$ 
[3] J.K. Webb, V.V. Flambaum, C.W. Churchill, M.J. Drinkwater and J.D. Barrow, Phys. Rev. Lett. 82, 884 (1999) arXiv:astro-ph/9803165;

S.K. Lamoreaux and J.R. Torgerson, Phys. Rev. D 69, 121701(R) (2004).

[4] E. Reinhold, R. Buning, U. Hollenstein, A. Ivanchik, P. Petitjean and W. Ubachs, Phys. Rev. Lett. 96, 151101 (2006).

[5] X. Calmet and H. Fritzsch, Eur. Phys. J. C 24, 639 (2002) arXiv:hep-ph/0112110;

P. Langacker, G. Segre and M.J. Strassler, Phys. Lett. B 528, 121 (2002) arXiv:hep-ph/0112233.

[6] R. Quast, D. Reimers and A. Levshakov, Astron. Astrophys. 415, L7 (2004) arXiv:astro-ph/0311280;

R. Srianand, H. Chand, P. Petitjean and B. Aracil, Phys. Rev. Lett. 92, 121302 (2004) arXiv:astro-ph/0402177.

[7] K.A. Olive, M. Pospelov, Y.Z. Qian, A. Coc, M. Cassé and E. Vangioni-Flam, Phys. Rev. D 66, 045022 (2002) arXiv:hep-ph/0205269.

[8] J.G. Williams, S.G. Turyshev and D.H. Boggs, Phys. Rev. Lett. 93, 261101 (2004) arXiv:gr-qc/0411113.

[9] C. Bambi, M. Giannotti and F.L. Villante, Phys. Rev. D 71, 123524 (2005) arXiv:astro-ph/0503502.

[10] V.M. Kaspi, J.H. Taylor and M.F. Ryba, Astrophys. J. 428, 713 (1994).

[11] S.E. Thorsett, Phys. Rev. Lett. 77, 1432 (1996) arXiv:astro-ph/9607003.

[12] P. Jofré, A. Reisenegger and R. Fernández, Phys. Rev. Lett. 97, 131102 (2006) arXiv:astro-ph/0606708.

[13] J. Garcia-Bellido, Int. J. Mod. Phys. D 2, 85 (1993) arXiv:hep-ph/9205216.

[14] S.L. Saphiro and S.A. Teukolsky, Black Holes, White Dwarfs, and Neutron Stars: The Physics of Compact Objects (Wiley, New York, USA, 1983).

[15] N.K. Glendenning, Compact Stars (Springer, New York, USA, 1997).

[16] C. Bambi, JCAP 0706, 006 (2007) arXiv:0704.2126 [hep-ph]].

[17] C. Bambi, Int. J. Mod. Phys. D (in press)
arXiv:0710.2042 [gr-qc]].

[18] M.A. Stephanov, PoS LAT2006, 024 (2006) arXiv:hep-lat/0701002.

[19] I. Bombaci and B. Datta, Astrophys. J. 530, L69 (2000) arXiv:astro-ph/0001478;

A. Drago, A. Lavagno and G. Pagliara, Phys. Rev D 69, 057505 (2004) arXiv:nucl-th/0401052.

[20] R. Ouyed and F. Sannino, Astron. Astrophys. 387, 725 (2002) arXiv:astro-ph/0103022;

Z. Berezhiani, I. Bombaci, A. Drago, F. Frontera and A. Lavagno, Astrophys. J. 586, 1250 (2003) arXiv:astro-ph/0209257;

A.G. Aksenov, M. Milgrom and V.V. Usov, Mon. Not. Roy. Astron. Soc. 343, L69 (2003) arXiv:astro-ph/0306361.

[21] P. Meszaros, Rept. Prog. Phys. 69, 2259 (2006) arXiv:astro-ph/0605208.

[22] A. Chodos, R.L. Jaffe, K. Johnson, C.B. Thorn and V.F. Weisskopf, Phys. Rev. D 9, 3471 (1974);

T. DeGrand, R.L. Jaffe, K. Johnson and J. Kiskis, Phys. Rev. D 12, 2060 (1975).

[23] M. Buballa, Phys. Rept. 407, $205 \quad$ (2005) arXiv:hep-ph/0402234.

[24] A. Akmal, V.R. Pandharipande and D.G. Ravenhall, Phys. Rev. C 58, 1804 (1998) arXiv:nucl-th/9804027.

[25] D. Guetta, T. Piran and E. Waxman, Astrophys. J. 619, 412 (2005) arXiv:astro-ph/0311488.

[26] M.D. Kistler, H. Yuksel, J.F. Beacom and K.Z. Stanek, arXiv:0709.0381.

[27] Indeed, the number of stars in a general galaxy can be estimated to be about $10^{11}$, while the number of galaxies in the visible universe is roughly $10^{12}$.

[28] Here, as well as in Eq. (12) below, the temporal variation is respect to the cosmological time, that is the time coordinate of the metric describing the expanding universe. It must be so because the prediction of time varying "fundamental constants" arises when we consider cosmological solutions of theories beyond general relativity. 